\title{
Simultaneous dimerization and SU(4) symmetry breaking of 4-color fermions on the square lattice
}

\author{
Philippe Corboz, ${ }^{1,2}$ Andreas M. Läuchli,,${ }^{3,4}$ Karlo Penc, ${ }^{5}$ Matthias Troyer, ${ }^{1}$ and Frédéric Mila ${ }^{2}$ \\ ${ }^{I}$ Theoretische Physik, ETH Zurich, 8093 Zurich, Switzerland \\ ${ }^{2}$ Institut de théorie des phénomènes physiques, École Polytechnique Fédérale de Lausanne, CH-1015 Lausanne, Switzerland \\ ${ }^{3}$ Max-Planck-Institut für Physik komplexer Systeme, Nöthnitzer Straße 38, D-01187 Dresden, Germany \\ ${ }^{4}$ Institut für Theoretische Physik, Universität Innsbruck, A-6020 Innsbruck, Austria \\ ${ }^{5}$ Research Institute for Solid State Physics and Optics, H-1525 Budapest, P.O. Box 49, Hungary
}

(Dated: February 13, 2018)

\begin{abstract}
Using infinite projected entangled pair states (iPEPS), exact diagonalization, and flavor-wave theory, we show that the SU(4) Heisenberg model undergoes a spontaneous dimerization on the square lattice, in contrast to its SU(2) and SU(3) counterparts, which develop Néel and three-sublattice stripe-like long-range order. Since the ground state of a dimer is not a singlet for SU(4) but a 6-dimensional irrep, this leaves the door open for further symmetry breaking. We provide evidence that, unlike in SU(4) ladders, where dimers pair up to form singlet plaquettes, here the SU(4) symmetry is additionally broken, leading to a gapless spectrum in spite of the broken translational symmetry.
\end{abstract}

PACS numbers: 67.85.-d, 71.10.Fd, 75.10.Jm, 02.70.-c

The search for new quantum phases of matter, one of the main themes in the field of electronic materials with strong correlations, has become an important aspect of the physics of cold atoms too. A recent development concerns the possibility of loading multi-color fermions in optical lattices, which has reopened the investigation of the $\mathrm{SU}(N)$ Heisenberg model that describes the permutation of $N$-color objects on a lattice [1-4]. In condensed matter, these models require fine tuning of parameters. For instance, the SU(3) Heisenberg model is realized in spin-1 models with equal bilinear and biquadratic interactions, while in standard antiferromagnetic materials the biquadratic interaction is much smaller than the bilinear one. In the same spirit, the SU(4) model can be seen as a symmetric version of the spin-orbital Kugel-Khomskii model [5], but in actual materials the orbital-orbital interaction is not rotationally invariant [6]. By contrast, the simple quantum permutation embodied by the $\mathrm{SU}(N)$ Heisenberg model is a realistic starting point to describe the Mott phase of $N$-color fermions at filling $1 / N$ (one particle per site).

In this paper, motivated by the conflicting results published so far, we concentrate on the case of 4-color fermions on the square lattice. In the Mott insulating phase with one fermion per site and large on-site repulsion, the relevant effective model is defined by the Hamiltonian

$$
\mathcal{H}=J \sum_{\langle i, j\rangle} P_{i j},
$$

where the transposition operator $P_{i j}$ exchanges two colors on neighboring sites, and $J>0$.

An investigation of the model based on a variational and mean-field study suggested a plaquette ground state [7], without a long-range color-order. Exact diagonalizations of 8 and 16 site clusters reported the presence of low lying singlets [9], and, inspired by the spontaneous singlet plaquette formation in the SU(4) ladder [10], suggested that these low-lying singlets might live in the subspace of singlet plaquette coverings, with possibly plaquette long-range order [8]. A different con-

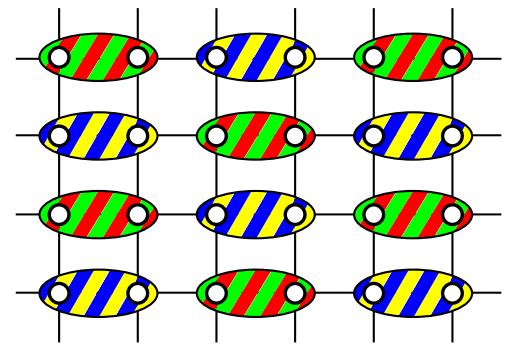

FIG. 1: (Color online) Sketch of dimer and color order realized in the ground state of the SU(4) Heisenberg model on the square lattice.

clusion has been reached in Ref. 11, where a gapless spin liquid with nodal fermions has been shown to be the variational ground state for a class of projected fermionic wave functions. The presence of a long-range color order with wave-vector $(2 \pi / N, 2 \pi / N)$ for $N=2$ (Néel order) and $N=3$ (threesublattice order with diagonal stripes [13]) hints to the possibility of extending such an ordering to $N=4$. Lastly, a chiral spin liquid is predicted based on a particular large-N limit, but not below $\mathrm{N}=5$ [12].

In this Letter, we show that none of these possibilities is realized, and that in fact both spatial and SU(4) symmetries are simultaneously broken: the ground state is spontaneously dimerized, and on each strong dimer, two colors dominate and combine into a 6-dimensional irrep of SU(4). In the resulting pattern, the same color does not appear on neighboring dimers, so that if two colors are dominant on one dimer, nearest-neighbor dimers are dominated by the remaining two colors, resulting in the Néel-like arrangement sketched in Fig. 1.

The first hint that simple color ordering is probably not realized appears at the level of linear flavor-wave theory (LFWT), the very method that confirmed the presence of stripe-order in the SU(3) case [13]. The starting point is a Hartree approximation based on a site-factorized variational wave function. 

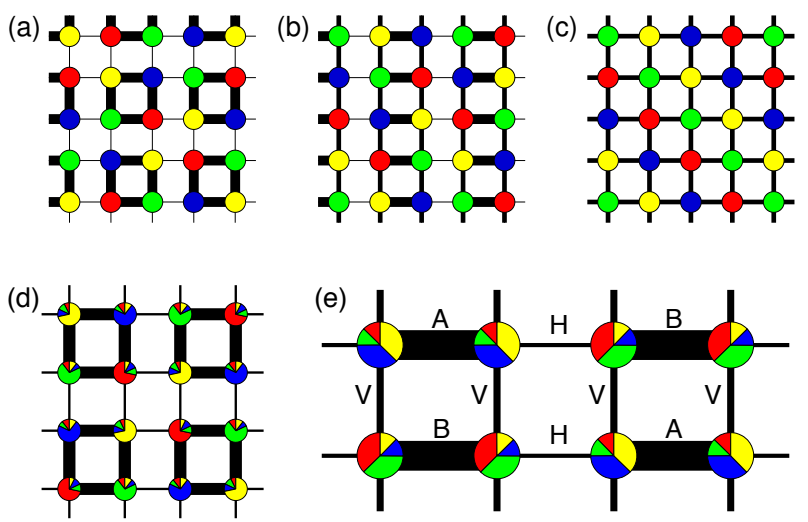

FIG. 2: (Color online) Sketch of some color and bond-energy configurations found by LFWT (a-c) and iPEPS (d-e). In all plots, the thickness of the lines is proportional to the square of $\left\langle P_{i j}\right\rangle$ on the corresponding bond. The color content reflects that of the Hartree starting point for LFWT, and the actual result for an appropriate, infinitesimal symmetry breaking field for iPEPS. (a) 'Plaquette' state selected by quantum fluctuations within LFWT. Its energy per site $E_{0} / N=-3 J / 2$. (b) 'Dimerized' state with LFWT energy per site $E_{0} / N=-1.293 J$. (c) Stripe-ordered state, with much higher LFWT energy per site $E_{0} / N=(4 / \pi-2) J \simeq-0.727 J$. (d) iPEPS results with $D=2$ and a unit cell $4 \times 4$. At this order, the bondenergy pattern of the ground state is similar to that of the plaquette state selected by LFWT. (e) iPEPS results for $D=12$ and a unit cell $4 \times 2$. The bond-energy pattern of this state is similar to that of the dimerized state of LFWT. Two types of dimers A and B with energy much lower than vertical (V) and horizontal (H) dimers can be identified. They differ from each other by their local color densities.

At this level, any state with different nearest-neighbor colors minimizes the energy, and, as in the case of SU(3), there is a macroscopic large number of such states. The next level of approximation consists in including quantum fluctuations within LFWT: The classical energy of each state is corrected by the zero point energy of a quadratic bosonic Hamiltonian which, on a nearest-neighbor bond with color $a$ on site $i$ and color $b$ on site $j$, is given by $\mathcal{H}_{\mathrm{fw}}=A_{i j}^{\dagger} A_{i j}-1$, with $A_{i j}^{\dagger}=a_{j}^{\dagger}+b_{i}$ where $a_{j}$ and $b_{i}$ are bosonic operators. Since there is an infinite number of Hartree ground states, we have used the following strategy: compare simple, periodic states, and make a systematic exploration of all states on finite clusters. The resulting picture is summarized in Fig. 2(a-c). First of all, and most importantly, the state with stripe order of Fig. 2(c), which breaks SU(4) symmetry but leaves all nearest neighbor bonds equivalent, is clearly not favored. In fact, many periodic states which break the spatial symmetry between bonds concomitantly with the SU(4) symmetry have much lower energy, the lowest one being a quadrumerized state in which strong bonds build 4-site plaquettes (Fig. 2(a)). Among possible states we can also find a dimerized state (Fig. 2(b)) with energy slightly above that of the plaquette state but far below that of the stripe state.

This result, rather surprising in view of the SU(3) case [13], can be rationalized as follows: i) In a given ground state, the
LFWT Hamiltonian is the sum of independent Hamiltonians that describe the motion of bosons on the connected clusters spanned by pairs of colors. For example, if a pair of neighboring sites with say colors $a$ and $b$ is such that all nearest neighbor sites have colors different from $a$ and $b$, it forms a 2site connected cluster. For the state of Fig. 2(a), there are two types of clusters: 2-site clusters on strong bonds, and 4-site clusters (plaquettes) on weak bonds; ii) The zero point energy per bond tends to increase with the cluster size. In particular, on a 2-site cluster, the ground state energy of the Hamiltonian is equal to -1 , i.e. there is no zero-point contribution to the energy[14], whereas larger clusters lead to finite frequencies, hence to strictly positive contributions to the zero-point energy. In the stripe-ordered state of Fig. 2(c), all clusters are infinite, one dimensional zig-zag stripes, and no bond is able to minimize the energy. By contrast, half the bonds have energy -1 in the state of Fig. 2(a) (thick bonds) and a quarter of them in the dimer state of Fig. 2(b). The difference betweeen the present case and $\mathrm{SU}(3)$ comes from the fact that it is impossible to realize isolated two-site clusters with only three colors on the square lattice, hence to have local bonds with very low energy.

So flavor-wave theory gives strong indication that the ground state is not a simple color-ordered state, and that some kind of additional lattice symmetry breaking takes place. One may be tempted to go one step further, and to conclude that it predicts plaquette order. However, if the symmetry is broken and small clusters appear, LFWT becomes inaccurate (for instance, it cannot restore the SU(4) symmetry in the ground states of a 4-site cluster), and one should rather turn to alternative methods.

Infinite projected entangled-pair states (iPEPS) results Next, we present results obtained with iPEPS [15], generalized to arbitrary unit cells [16], a variational approach based on a tensor network ansatz aimed at efficiently representing the ground state of two-dimensional lattice models in the thermodynamic limit. It consists of a network of rank- 5 tensors, one for each lattice site, where each tensor is connected to its nearest neighbors by four bond indices with a certain bond dimension $D$, and the fifth index carries the local Hilbert space of a lattice site. The accuracy of the ansatz can be systematically controlled by the bond-dimension $D$. We use $N_{T}$ different tensors, arranged in a rectangular unit cell of size $L_{x} \times L_{y}=N_{T}$ which is periodically repeated in the lattice [16]. The tensors are optimized by performing an imaginary time evolution, using the so-called simple update $[17,18]$. For the computation of expectation values we use the cornertransfer matrix method $[16,20]$ to approximately contract the tensor network, where the accuracy of the contraction can be controlled by another parameter called boundary dimension $\chi$. The simulation results presented in the following are extrapolated in $\chi$, where the extrapolation error is small compared to the symbol sizes. To improve the efficiency for large bond dimensions we use tensors with $\mathbb{Z}_{q}$ symmetry [21] [a discrete subgroup of $\mathrm{SU}(4)$ ].

Figure 3(a) shows the iPEPS results for the energy per site 

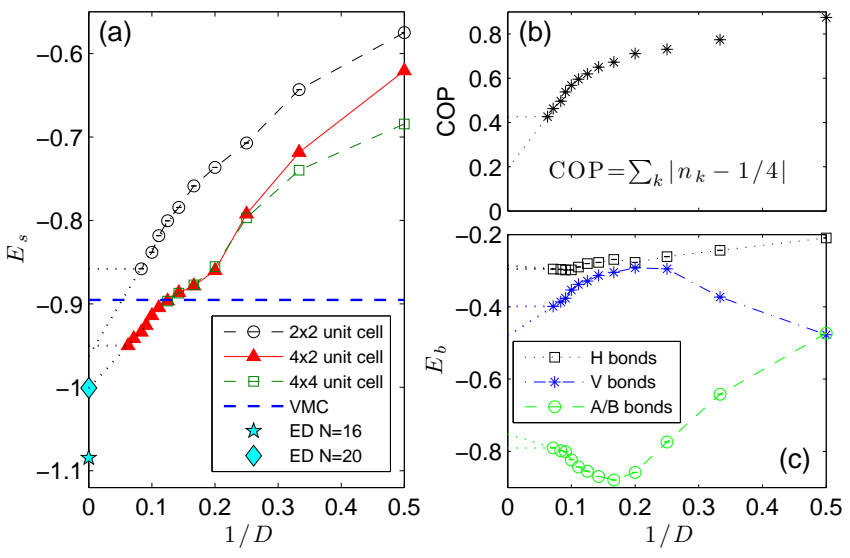

FIG. 3: (Color online) iPEPS results as a function of the inverse bond dimension $1 / D$. Dotted lines are only a guide to the eye. (a) Energy per site in units of $J$ for different unit cell sizes in the iPEPS, compared to the variational Monte Carlo (VMC) energy from Ref. [11] (extrapolated to the thermodynamic limit), and exact diagonalization results. (b) Color order parameter (COP), which is zero if on each site the color density $n_{k}$ of each color $k$ is the same, i.e. $n_{k}=0.25$. The data does not seem to extrapolate to zero in the limit $D \rightarrow \infty$ and we therefore expect the SU(4) symmetry to be spontaneously broken. (c) Bond energies of the bonds $\mathrm{H}, \mathrm{V}, \mathrm{A}$ and $\mathrm{B}$ marked in Fig. 2 obtained from the $4 \times 2$ unit cell for $D>4$ and from the $4 \times 4$ unit cell for $D \leq 4$. The dimerization (strong $\mathrm{A} / \mathrm{B}$ bonds) is seen to persist in the limit $D \rightarrow \infty$.

as a function of $1 / D$ for different unit cell sizes. The energy obtained with the $2 \times 2$ unit cell is considerably higher than the one obtained with the $4 \times 2$ and $4 \times 4$ unit cells. For $D \leq 4$ the lowest energy is obtained with the $4 \times 4$ unit cell, while for $D>4$ similar results are obtained with the $4 \times 2$ unit cell. This indicates that the ground state breaks translational invariance in a way which is compatible with the $4 \times 2$ unit cell, but not with the $2 \times 2$ unit cell. For a bond dimension $D=8$ the iPEPS variational energy is comparable to the variational Monte Carlo (VMC) energy from Ref. [11], however, for larger $D$ we obtain energies which are considerably lower. We note that the energy has not converged yet with bond dimension $D$, and thus further corrections to the ground state energy (and other quantities) can be expected for larger $D$.

We next turn our focus to local properties of the ground state. The bottom part of Fig. 2 shows the local color densities on each site and the energy on each bond for $D=2$ (left) and $D=12$ (right). The evolution with $D$ of the energy of the various bonds is shown in Fig. 3(c). For $D=2$, the bond pattern is similar to that of the linear FWT groundstate of Fig. 2(a). However, as soon as $D>2$, three types of bonds appear in the ground state, and the bonds form a columnar dimerized pattern similar to the linear FWT of Fig. 2(b). Since increasing $D$ allows more quantum fluctuations, we interpret this result as an indication that the dimerized pattern is ultimately stabilized when enough quantum fluctuations are taken into account.

For $\mathrm{SU}(2)$ antiferromagnets, if spontaneous formation of dimers occurs, this is the end of the story: The ground state degeneracy is given by the number of equivalent dimer coverings, and each ground state is adiabatically connected to a product of singlets on the dimers. However, for SU(4), it takes at least 4 sites to make a singlet, and on a dimer, the ground state is 6-fold degenerate. This 6-dimensional subspace corresponds to the 6-dimensional irrep of SU(4). A convenient basis is provided by the states $(|\alpha \beta\rangle-|\beta \alpha\rangle) / \sqrt{2}$ where the couple $(\alpha, \beta)$ is a pair of different colors chosen out of the colors of the model and can take 6 values. These states are connected by SU(4) rotations. So, even in the presence of spontaneous dimerization, there is room for further SU(4) symmetry breaking. In our iPEPS results for $D=12$, one can immediately recognize the formation of two types of dimers, marked with A and B in Fig. 2(e), which are arranged in a (columnar) checkerboard order. The energy of both A and B dimers is identical, however, the local color densities are different. In dimers A the first two colors [blue and yellow in Fig. 2(e)] are dominant, whereas in the B-dimers the density of the other two colors is larger. [Here we applied a small initial field to fix the "direction" of the SU(4) symmetry breaking in the space of the four colors. We note that the same results (up to SU(4) rotations) are obtained without an initial field]. This type of order is seen to persist also in the limit $D \rightarrow \infty$, as shown in Fig. 3(b). We also verified that in the simulations done with a $4 \times 4$ unit cell this pattern is repeated twice. Thus, our iPEPS results suggest that both the SU(4) symmetry and translational symmetry are broken, resulting in a Néel-like order with dimers alternating between pairs of colors.

Exact diagonalization $(E D)$ results - We now compare these predictions with the results of ED of finite clusters. The energies per site $[E /(N J)]$ in the ground state are -1 for $N=8,-1.0844$ for $N=16$ [9] and -1.0008 for $N=20$. In Fig. 4 we present results for several correlation functions obtained for the $N=16$ system. In Fig. 4(a) Fouriertransformed color-density correlations are shown. The diameters of the circles are proportional to the Fourier component. The maxima are found at momentum $(\pi, \pi / 2)$ and equivalent positions. This provides a possible explanation as to why the $N=8$ and $N=20$ samples have higher energy per site than the $N=16$ cluster, as their Brillouin zone does not contain these particular momenta and thus frustrate the preferred order [22]. In Fig. 4(b) we display the connected nearestneighbor bond energy correlations $\left\langle P_{i j} P_{k l}\right\rangle-\left\langle P_{i j}\right\rangle^{2}$. The correlations present a clear striped signal and are in qualitative agreement with the bond energy modulation pattern obtained using iPEPS, however the modulation is not very strong. Finally in Fig. 4(c) we calculate correlations between the projectors onto a given basis state of the $d=6$ representation of $\mathrm{SU}(4)$ on a bond $\left\langle\mathcal{P}_{(a, b)}(i, j) \mathcal{P}_{(a, b)}(k, l)\right\rangle$. This correlation supposedly captures the Néel structure on top of a columnar dimerized background. Indeed the correlations in (c) show the expected four positively correlated bonds at the correct location confirming the qualitative picture put forward in Fig. 1. We have also measured the spin chirality correlations advocated in Ref. [12]. We indeed find correlations pointing to- 

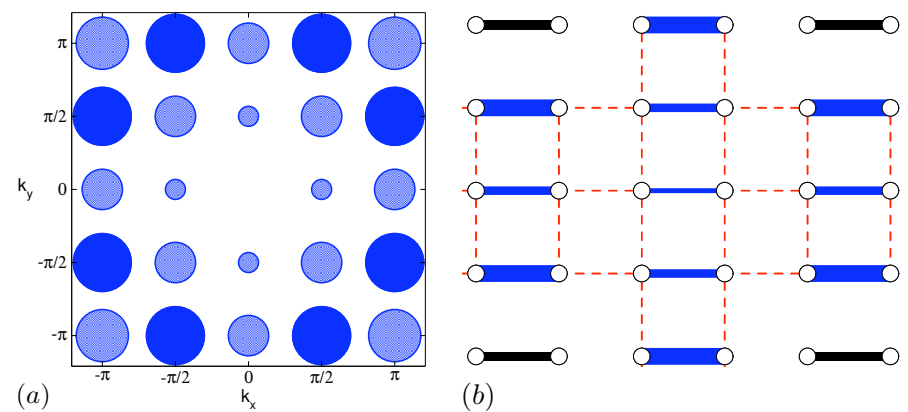

(b)

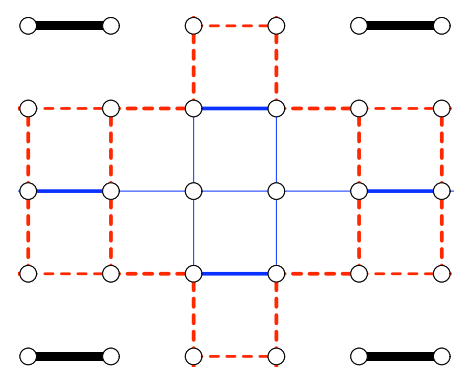

$(c)$

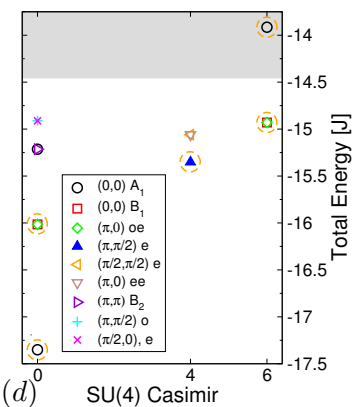

$(d)^{0} \quad \mathrm{SU}(4)$ Casimir

FIG. 4: (Color online) (a) Color structure factor for the $N=16$ square sample in momentum space. The maxima of the structure factor (filled circles) are located at momentum $(\pi, \pi / 2)$ and equivalent points. (b) Connected bond energy correlations $\left\langle P_{i j} P_{k l}\right\rangle-\left\langle P_{i j}\right\rangle^{2}$ for $N=16$. (c) $\left\langle\mathcal{P}_{(a, b)}(i, j) \mathcal{P}_{(a, b)}(k, l)\right\rangle$. In (b) and (c) the line width is proportional to the absolute value of the correlations and blue, full (red, dashed) lines denote positive (negative) values. (d) Low energy spectrum of the $N=16$ sample plotted as a function of the quadratic SU(4) Casimir operator $C$. Expected levels in the tower of states of the SU(4) symmetry breaking state sketched in Fig. 1 are highlighted by dashed circles. In the grey shaded region there are additional levels (not shown) which have not been resolved as function of the Casimir.

wards uniform chirality on triangles, however the correlations decay quickly over the size of the sample, implying the probable absence of a chiral spin liquid phase on the SU(4) square lattice.

We conclude this ED section by presenting the low energy tower of state in Fig. 4(d) as a function of the quadratic SU(4) Casimir operator. In the case of either discrete or continuous symmetry breaking the low energy spectrum is expected to exhibit characteristic energy levels which become degenerate with the ground state in the thermodynamic limit and which enable the spontaneous symmetry breaking. For the proposed state in Fig. 1 we expect a tower of states which is a fourfold superposition of the tower of state of a SU(4) 6 bipartite Néel state. The later is given by the irrep content $C=0,4,6,10,12, \ldots$. Combining with the spatial degeneracy associated with the four distinct dimer configurations, the even members of the Casimir sequence are expected to belong to the spatial symmetry sectors $(0,0) A_{1},(0,0) B_{1}$ and the twofold degenerate sector $(\pi, 0) \sigma_{x}=-1, \sigma_{y}=1$, while the odd members belong to the fourfold degenerate momentum $(\pi, \pi / 2) \sigma_{x}=1$ sector. When comparing this prediction (large circles) to the actual low energy spectrum shown in Fig. 4(d) one observes that the lowest levels for $C=0$ and 4 are indeed the ones expected for the proposed state in Fig. 1.

Conclusion - The various approaches followed in this paper point to a rather coherent picture: a spontaneous dimerization appears in the ground state, and the remaining degrees of freedom, an irrep of dimension 6 on each dimer, develop Néel-like order. The model that describes the fluctuations around one of the dimerized ground states should thus be a model with SU(4) symmetry with the 6-dimensional irrep at each site of a square lattice and with anisotropic couplings horizontal and vertical pairs of nearest-neighbor dimers are inequivalent. In that respect, it is interesting to note that the isotropic version of the SU(4) Heisenberg model with the 6dimensional irrep and only nearest-neighbor couplings has been studied by Quantum Monte Carlo [23] and variational
Monte Carlo [24] with conflicting conclusions. While in the latter study long-range Néel order is found, the former predicts that the correlations decay algebraically, i.e. that there is no true long-range order. Coming back to the evidence in favor of Néel order in our case, the presence of short-range correlations is clear in view of the ED and iPEPS results. However, correlations revealed by ED are not very strong, and the prediction of long-range order relies mostly on the empirical $1 / D$ scaling of the iPEPS order parameter of Fig. 3. It would thus be very interesting to challenge this prediction by deriving and studying the effective model relevant to the present case.

We acknowledge the financial support of the Swiss National Fund and of MaNEP, and of the Hungarian OTKA Grant No. K73455. The simulations were performed on the Brutus cluster at ETH Zurich and on the PKS-AIMS cluster at the MPG RZ Garching.

[1] C. Wu, J. P. Hu, and S. C. Zhang, Phys. Rev. Lett. 91, 186402 (2003).

[2] C. Honerkamp, and W. Hofstetter, Phys. Rev. Lett. 92, 170403 (2004).

[3] M. A. Cazalilla, A. F. Ho, and M. Ueda, New J. Phys. 11, 103033 (2009)

[4] A. V. Gorshkov, M. Hermele, V. Gurarie, C. Xu, P. S. Julienne, J. Ye, P. Zoller, E. Demler, M. D. Lukin, and A. M. Rey, Nat. Phys. 6, 289 (2010).

[5] K. I. Kugel and D. I. Khomskii, Sov. Phys. Usp. 25, 231 (1982).

[6] The $d$-electron system $\mathrm{LiNiO}_{2}$ [25] and the $f$-electron system $\mathrm{CeB}_{6}[26]$ are believed to be quite close to an SU(4) symmetric model.

[7] Y. Q. Li, Michael Ma, D. N. Shi, and F. C. Zhang, Phys. Rev. Lett. 81, 3527 (1998).

[8] H.-H. Hung, Y. Wang, and C. Wu, Phys. Rev. B 84, 054406 (2011).

[9] M. van den Bossche, F.-C. Zhang, and F. Mila, Eur. Phys. J. B 17, 367 (2000). 
[10] M. van den Bossche, P. Azaria, P. Lecheminant, and F. Mila, Phys Rev. Lett. 86, 4124 (2001).

[11] F. Wang and A. Vishwanath, Phys. Rev. B 80, 064413 (2009).

[12] M. Hermele, V. Gurarie, and A. M. Rey, Phys. Rev. Lett. 103, 135301 (2009).

[13] T. A. Tóth, A. M. Läuchli, F. Mila, and K. Penc, Phys. Rev. Lett. 105, 265301 (2010).

[14] On two sites, the Bogoliubov transformation is singular because $\left[A_{i j}^{\dagger}, A_{i j}\right]=0$. However, the ground state energy can be shown to be equal to -1 , and the zero point energy to vanish because the frequencies of the two underlying harmonic oscillators are equal to zero. A pair of zero frequency modes is actually present on all finite clusters.

[15] F. Verstraete and J. I. Cirac, cond-mat/0407066. V. Murg, F. Verstraete, and J. I. Cirac, Phys. Rev. A 75, 033605 (2007). G. Sierra and M. A. Martín-Delgado, e-print arXiv:condmat/9811170; Y. Nishio, N. Maeshima, A. Gendiar, and T. Nishino, e-print arXiv:cond-mat/0401115; J. Jordan, R. Orús, G. Vidal, F. Verstraete, and J. I. Cirac, Phys. Rev. Lett. 101, 250602 (2008).

[16] P. Corboz, S. R. White, G. Vidal, and M. Troyer, Phys. Rev. B 84, 041108(R) (2011)

[17] H. C. Jiang, Z. Y. Weng, and T. Xiang, Phys. Rev. Lett. 101, 090603 (2008).

[18] We verified our results up to a bond-dimension $D=8$ with the so-called full-update (cf. [19]), which is optimal but computa- tionally more expensive.

[19] P. Corboz, R. Orús, B. Bauer, and G. Vidal, Phys. Rev. B 81, 165104 (2010).

[20] R. J. Baxter, J. Math. Phys. 9, 650 (1968); J. Stat. Phys. 19, 461 (1978); Exactly Solved Models in Statistical Mechanics (Academic Press, London, 1982); T. Nishino and K. Okunishi, J. Phys. Soc. Jpn. 65, 891 (1996); R. Orús and G. Vidal, Phys. Rev. B 80, 094403 (2009); P. Corboz, J. Jordan, and G. Vidal. Phys. Rev. B 82, 245119 (2010).

[21] S. Singh, R. N. C. Pfeifer, and G. Vidal, Phys. Rev. B 83, 115125 (2011); B. Bauer, P. Corboz, R. Orús, and M. Troyer, Phys. Rev. B 83, 125106 (2011).

[22] The $N=8$ sample is a periodic square cell spanned by the two vectors $\mathbf{T}_{1}=(2,2)$ and $\mathbf{T}_{2}=(-2,2) . N=16$ is spanned by $\mathbf{T}_{1}=(4,0)$ and $\mathbf{T}_{2}=(0,4)$ and $N=20$ is spanned by $\mathbf{T}_{1}=(4,2)$ and $\mathbf{T}_{2}=(-2,4)$.

[23] F. F. Assaad, Phys. Rev. B 71, 075103 (2005).

[24] A. Paramekanti and J. B. Marston, J. Phys.: Condens. Matter 19, 125215 (2007).

[25] Y. Kitaoka, T. Kobayashi, A. Koda, H. Wakabayashi, Y. Niino, H. Yamakage, S. Taguchi, K. Amaya, K. Yamaura, M. Takano, A. Hirano, and R. Kanno, J. Phys. Soc. Jpn. 67, 3703 (1998).

[26] R. Shiina, H. Shiba, and P. Thalmeier, J. Phys. Soc. Jpn. 66, 1741 (1997). 